\title{
Asymptotic behavior of the thermoelastic suspension bridge equation with linear memory
}

\section{Jum-Ran Kang ${ }^{*}$}

\section{"Correspondence:}

pointegg@hanmail.net Department of Mathematics, Dong-A University, Busan, 604-714, Korea

\begin{abstract}
This paper is concerned with a thermoelastic suspension bridge equations with memory effects. For the suspension bridge equations without memory, there are many classical results. However, the suspension bridge equations with both viscoelastic and thermal memories were not studied before. The object of the present paper is to provide a result on the global attractor to a thermoelastic suspension bridge equation with past history.
\end{abstract}

Keywords: global attractors; suspension bridge equation; viscoelasticity; memory; thermoelasticity; asymptotically compact

\section{Introduction}

In recent years, several authors have been concerned with the asymptotic behavior of the following suspension bridge equations:

$$
u_{t t}+\Delta^{2} u+k u^{+}+\delta u_{t}+f(u)=h(x)
$$

where $u(x, t)$ is an unknown function, which represents the deflection of the roadbed in the vertical plane, $k>0$ denotes the spring constant of the ties, and $\delta>0$ is a given constant. The force $u^{+}=\max \{u, 0\}$ is the positive part of $u$. The suspension bridge equations are an important mathematical model in engineering. Lazer and McKenna [1] investigated the problem of nonlinear oscillation in a suspension bridge. Lately, similar models have been considered by many authors, most of them concentrating on the existence and decay estimates of solutions; see [2-4] and references therein. Ma and Zhong [5] and Zhong et al. [6] proved the existence of global attractors of weak and strong solutions for equation (1.1), respectively. Park and Kang [7] showed the existence of pullback attractor for a nonautonomous suspension bridge equation with linear damping and in [8] obtained the existence of global attractors for the suspension bridge equations with nonlinear damping. Besides, the problem of attractor of the solutions to a coupled system of suspension bridge equations has been studied by several authors [9-12]. Recently, Kang [13] proved the longtime behavior to the suspension bridge equation when the unique damping mechanism is given by the memory term. We construct some proper Lyapunov functions to show the existence of global attractors. The asymptotic behavior of a thermoelastic system has

(c) Kang 2016. This article is distributed under the terms of the Creative Commons Attribution 4.0 International License (http://creativecommons.org/licenses/by/4.0/), which permits unrestricted use, distribution, and reproduction in any medium, provided you give appropriate credit to the original author(s) and the source, provide a link to the Creative Commons license, and indicate if changes were made. 
been widely investigated by many authors. In particular, the stability of a thermoelastic system with memory was proved by several authors [14-17]. To the best of our knowledge, problem (1.1) was not earlier considered in a thermoelasticity point of view. Since thermal effect is a major feature in the theory of elastic plates, we intend to investigate the dynamical behavior of a thermoelastic version of problem (1.1). This paper is concerned with long-time behavior of a solution to the following thermoelastic suspension bridge equation with linear memory:

$$
\begin{aligned}
& u_{t t}+\alpha \Delta^{2} u-\Delta u_{t}+k u^{+}-\int_{0}^{\infty} \mu(s) \Delta^{2} u(t-s) d s+\beta \Delta \theta+f(u) \\
& =h(x) \text { in } \Omega \times \mathbb{R}^{+}, \\
& \theta_{t}-\Delta \theta-\beta \Delta u_{t}-\int_{0}^{\infty} \kappa(s) \Delta \theta(t-s) d s=0 \quad \text { on } \Omega \times \mathbb{R}^{+}, \\
& u=0, \quad \Delta u=0, \quad \theta=0 \quad \text { on } \partial \Omega \times \mathbb{R}^{+}, \\
& u(x, t)=u_{0}(x, t), \quad u_{t}(x, t)=u_{1}(x, t), \\
& \theta(x, t)=\theta_{0}(x, t), \quad(x, t) \in \Omega \times(-\infty, 0],
\end{aligned}
$$

where $\Omega$ is a bounded domain in $\mathbb{R}^{2}$ with sufficiently smooth or rectangular boundary $\partial \Omega$, and $\Delta$ denotes the Laplace operator. Here $\alpha$ is the flexural rigidity of the structure, and $\beta>0$ provides connection between deflection and temperature and depends on mechanical and thermal properties of the material. The initial conditions $u_{0}, \theta_{0}: \Omega \times(-\infty, 0] \rightarrow \mathbb{R}$ are the prescribed past histories of $u$ and $\theta$, respectively. It is well known that $u=u(x, t)$ represents the deflection of the roadbed in the vertical plane and $\theta=\theta(x, t)$ is the temperature difference with respect to a fixed reference temperature. Memory kernels $\mu(s)$ and $\kappa(s)$ are supposed to be smooth decreasing convex functions vanishing at infinity.

The only way to associate a process with such equations is to view the past history of $u$ and $\theta$ as new variables of the system, which will be ruled by a supplementary equation. To formulate system (1.2)-(1.5) in a history space setting, as in [18-21], we define new variables $\eta$ and $\zeta$ by

$$
\eta^{t}(x, s)=u(x, t)-u(x, t-s), \quad \zeta^{t}(x, s)=\int_{0}^{s} \theta(x, t-y) d y, \quad(x, s) \in \Omega \times \mathbb{R}^{+}, t \geq 0 .
$$

Formally, they satisfy the linear equations

$$
\eta_{t}^{t}(x, s)+\eta_{s}^{t}(x, s)=u_{t}(x, t), \quad \zeta_{t}^{t}(x, s)+\zeta_{s}^{t}(x, s)=\theta(x, t), \quad(x, s) \in \Omega \times \mathbb{R}^{+}, t \geq 0,
$$

and

$$
\eta^{t}(x, 0)=0, \quad \zeta^{t}(x, 0)=0, \quad x \in \Omega, t \geq 0,
$$

whereas

$$
\eta^{0}(x, s)=u_{0}(x, 0)-u_{0}(x,-s), \quad \zeta^{0}(x, s)=\int_{0}^{s} \theta_{0}(x,-y) d y, \quad(x, s) \in \Omega \times \mathbb{R}^{+} .
$$


Assuming that $\mu, v \in L^{1}\left(\mathbb{R}^{+}\right)$and taking $\alpha=1+\int_{0}^{\infty} \mu(s) d s$ and $v(s)=-\kappa^{\prime}(s)$, problem (1.2)-

(1.5) can be transformed into the equivalent system

$$
\begin{aligned}
& u_{t t}+\Delta^{2} u-\Delta u_{t}+k u^{+}+\int_{0}^{\infty} \mu(s) \Delta^{2} \eta^{t}(s) d s+\beta \Delta \theta+f(u)=h(x) \quad \text { in } \Omega \times \mathbb{R}^{+}, \\
& \theta_{t}-\Delta \theta-\beta \Delta u_{t}-\int_{0}^{\infty} v(s) \Delta \zeta^{t}(s) d s=0 \quad \text { on } \Omega \times \mathbb{R}^{+}, \\
& \eta_{t}^{t}+\eta_{s}^{t}=u_{t}, \quad(x, t, s) \in \Omega \times \mathbb{R}^{+} \times \mathbb{R}^{+}, \\
& \zeta_{t}^{t}+\zeta_{s}^{t}=\theta, \quad(x, t, s) \in \Omega \times \mathbb{R}^{+} \times \mathbb{R}^{+},
\end{aligned}
$$

with boundary conditions

$$
\begin{array}{lll}
u=\Delta u=0, & \theta=0 & \text { on } \partial \Omega \times \mathbb{R}^{+}, \\
\eta=\Delta \eta=0, & \zeta=0 & \text { on } \partial \Omega \times \mathbb{R}^{+} \times \mathbb{R}^{+},
\end{array}
$$

and initial conditions

$$
\begin{aligned}
& u(x, 0)=u_{0}(x), \quad u_{t}(x, 0)=u_{1}(x), \quad \theta(x, 0)=\theta_{0}(x), \\
& \eta^{t}(x, 0)=0, \quad \zeta^{t}(x, 0)=0, \quad \eta^{0}(x, s)=\eta_{0}(x, s), \quad \zeta^{0}(x, s)=\zeta_{0}(x, s),
\end{aligned}
$$

where

$$
\left\{\begin{array}{l}
u_{0}(x)=u_{0}(x, 0), \quad u_{1}(x)=\left.\partial_{t} u_{0}(x, t)\right|_{t=0}, \quad \theta_{0}(x)=\theta_{0}(x, 0), \quad x \in \Omega, \\
\eta_{0}(x, s)=u_{0}(x, 0)-u_{0}(x,-s), \quad \zeta_{0}(x, s)=\int_{0}^{s} \theta_{0}(x,-y) d y, \quad(x, s) \in \Omega \times \mathbb{R}^{+} .
\end{array}\right.
$$

Because $h$ is independent of time, the initial-boundary value problem (1.6)-(1.11) is in fact an autonomous dynamical system with respect to the unknown pair $\left(u(t), u_{t}(t), \theta(t), \eta^{t}, \zeta^{t}\right)$. In order to settle (1.2)-(1.5) in the framework of dynamical systems, we investigate modified equations (1.6)-(1.11). Indeed, it turns out that they are the same thing; to be more precise, the modified equations are in fact a generalization of the original equations. In the past years, the asymptotic behavior of viscoelastic equations with past history has been studied by many authors (see [22-27]).

We formulate our assumptions and results with respect to these new systems. The hypotheses and the well-posedness for the system (1.6)-(1.11) are presented in Section 2. Also, we give some notation and fundamental results of infinite-dimensional dynamical systems. In Section 3, we establish our main result on the existence of a compact global attractor.

\section{Preliminaries}

Now we introduce the Hilbert spaces that will be used in our analysis. Let

$$
V_{0}=L^{2}(\Omega), \quad V_{1}=H_{0}^{1}(\Omega), \quad V_{2}=H^{2}(\Omega) \cap H_{0}^{1}(\Omega)
$$

As usual, $(\cdot, \cdot)$ denotes the $L^{2}$-inner product, and $\|\cdot\|_{p}$ denotes the $L^{p}$-norm. We consider the history spaces $L_{\mu}^{2}\left(\mathbb{R}^{+} ; V_{2}\right)$ and $L_{v}^{2}\left(\mathbb{R}^{+} ; V_{1}\right)$ of measurable functions $\eta$ with values in $V_{2}$ 
or $V_{1}$, respectively, such that

$$
\|\eta\|_{\mu, V_{2}}^{2}=\int_{0}^{\infty} \mu(s)\|\Delta \eta(s)\|^{2} d s<\infty
$$

and

$$
\|\eta\|_{v, V_{1}}^{2}=\int_{0}^{\infty} v(s)\|\nabla \eta(s)\|^{2} d s<\infty .
$$

The following Cartesian product of Hilbert spaces will play the role of a phase space for the considered model:

$$
\mathcal{H}=V_{2} \times V_{0} \times V_{0} \times L_{\mu}^{2}\left(\mathbb{R}^{+} ; V_{2}\right) \times L_{v}^{2}\left(\mathbb{R}^{+} ; V_{1}\right)
$$

with the norm

$$
\|(u, v, \theta, \eta, \zeta)\|_{\mathcal{H}}^{2}=\|\Delta u\|^{2}+\|v\|^{2}+\|\theta\|^{2}+\|\eta\|_{\mu, V_{2}}^{2}+\|\zeta\|_{\nu, V_{1}}^{2} .
$$

Let $\lambda_{1}$ and $\lambda$ be the best constants in the Poincáre inequalities

$$
\lambda_{1}\|u\|^{2} \leq\|\nabla u\|^{2}, \quad \lambda\|u\|^{2} \leq\|\Delta u\|^{2},
$$

respectively.

We assume that $h \in L^{2}(\Omega)$ and the forcing term $f: \mathbb{R} \rightarrow \mathbb{R}$ satisfy

$$
f(0)=0, \quad|f(u)-f(v)| \leq k_{0}\left(1+|u|^{p}+|v|^{p}\right)|u-v|, \quad u, v \in \mathbb{R},
$$

where $k_{0}>0$ and $p>0$. This implies that $H^{2}(\Omega) \cap H_{0}^{1}(\Omega) \hookrightarrow L^{2(p+1)}(\Omega)$. Besides, we assume that, for some $k_{1} \geq 0$,

$$
-k_{1} \leq F(u) \leq f(u) u, \quad u \in \mathbb{R}
$$

where $F(z)=\int_{0}^{z} f(s) d s$.

In addition, with respect to the memory kernels $\mu(s), v(s) \geq 0$, we assume that

$$
\mu, v \in C^{1}\left(\mathbb{R}^{+}\right) \cap L^{1}\left(\mathbb{R}^{+}\right), \quad \int_{0}^{\infty} \mu(s) d s=\mu_{0}>0, \quad \int_{0}^{\infty} v(s) d s=v_{0}>0,
$$

and that there exist constants $k_{2}, k_{3}>0$ such that

$$
\mu^{\prime}(s) \leq-k_{2} \mu(s), \quad v^{\prime}(s) \leq-k_{3} v(s), \quad s \geq 0 .
$$

The well-posedness of problem (1.6)-(1.11) can be obtained by the Faedo-Galerkin method (see $[4,5,28])$. For the problem involving a memory term, we follow arguments from $[20,21]$.

Theorem 2.1 Under assumptions (2.2)-(2.5), we have 
(i) For every initial data $\left(u_{0}, u_{1}, \theta_{0}, \eta_{0}, \zeta_{0}\right) \in \mathcal{H}$, problem (1.6)-(1.11) has a weak solution

$$
\left(u, u_{t}, \theta, \eta, \zeta\right) \in C([0, T] ; \mathcal{H}), \quad T>0
$$

satisfying

$$
\begin{aligned}
& u \in L^{\infty}\left(0, T ; V_{2}\right), \quad u_{t}, \theta \in L^{\infty}\left(0, T ; V_{0}\right), \\
& \eta \in L^{\infty}\left(0, T ; L_{\mu}^{2}\left(\mathbb{R}^{+} ; V_{2}\right)\right), \quad \zeta \in L^{\infty}\left(0, T ; L_{v}^{2}\left(\mathbb{R}^{+} ; V_{1}\right)\right) .
\end{aligned}
$$

(ii) The weak solutions depend continuously on the initial data in $\mathcal{H}$. More precisely, given any two weak solutions $z_{1}, z_{2}$ of problem (1.6)-(1.11), we have

$$
\left\|z_{1}(t)-z_{2}(t)\right\|_{\mathcal{H}} \leq e^{c t}\left\|z_{1}(0)-z_{2}(0)\right\|_{\mathcal{H}^{\prime}} \quad t \in[0, T]
$$

for some constant $c>0$.

Remark 2.1 The well-posedness of problem (1.6)-(1.11) implies that the solution operator $S(t): \mathcal{H} \rightarrow \mathcal{H}$ defined by

$$
S(t)\left(u_{0}, u_{1}, \theta_{0}, \eta_{0}, \zeta_{0}\right)=\left(u(t), u_{t}(t), \theta(t), \eta^{t}, \zeta^{t}\right), \quad t \geq 0
$$

satisfies the semigroup properties and defines a nonlinear $C_{0}$-semigroup, which is locally Lipschitz continuous on $\mathcal{H}$. Thus, we can study (1.6)-(1.11) as a nonlinear dynamical system $(\mathcal{H}, S(t))$.

Now, we recall some fundamental results of infinite-dimensional dynamical systems (see [29-31]).

Definition 2.1 Let $S(t)$ be a $C_{0}$-semigroup defined in a Banach space $X$. A global attractor for $(X, S(t))$ is a bounded closed set $\mathcal{A} \subset X$ that is fully invariant and uniformly attracting, that is, $S(t) \mathcal{A}=\mathcal{A}$ for all $t>0$, and for every bounded subset $B \subset X$,

$$
\lim _{t \rightarrow \infty} \operatorname{dist}_{X}(S(t) B, \mathcal{A})=0
$$

where $\operatorname{dist}_{X}(Y, Z)=\sup _{y \in Y} \inf _{z \in Z} d(y, z)$ is the Hausdorff semidistance between $Y$ and $Z$ in $X$.

Definition 2.2 A dynamical system $(X, S(t))$ is dissipative if it possesses a bounded absorbing set, that is, a bounded set $\mathcal{B} \subset X$ such that, for any bounded set $B \subset X$, there exists $t_{B} \geq 0$ satisfying

$$
S(t) B \subset \mathcal{B}, \quad t \geq t_{B}
$$

Definition 2.3 Let $X$ be a Banach space, and $B$ be a bounded subset of $X$. We call a function $\phi(\cdot, \cdot)$ defined on $X \times X$ a contractive function on $B \times B$ if for any sequence $\left\{x_{n}\right\}_{n=1}^{\infty} \subset B$, there is a subsequence $\left\{x_{n_{k}}\right\}_{k=1}^{\infty} \subset\left\{x_{n}\right\}_{n=1}^{\infty}$ such that

$$
\lim _{k \rightarrow \infty} \lim _{l \rightarrow \infty} \phi\left(x_{n_{k}}, x_{n_{l}}\right)=0
$$


Theorem $2.2([30])$ Let $\{S(t)\}_{t \geq 0}$ be a semigroup on a Banach space $(X,\|\cdot\|)$ that has a bounded absorbing set $B_{0}$. Moreover, assume that, for any $\epsilon \geq 0$, there exist $T=T\left(B_{0}, \epsilon\right)$ and $\phi_{T}(\cdot, \cdot) \in C(B)$ such that

$$
\|S(T) x-S(T) y\| \leq \epsilon+\phi_{T}(x, y) \quad \text { for all } x, y \in B_{0},
$$

where $C(B)$ is a set of all contractive functions on $B \times B$, and $\phi_{T}$ depends on $T$. Then $\{S(t)\}_{t \geq 0}$ is asymptotically compact in $X$, that is, for any bounded sequence $\left\{y_{n}\right\}_{n=1}^{\infty} \subset X$ and any sequence $\left\{t_{n}\right\}$ with $t_{n} \rightarrow \infty,\left\{S\left(t_{n}\right) y_{n}\right\}_{n=1}^{\infty}$ is precompact in $X$.

Theorem 2.3 ([30]) A dissipative dynamical system $(X, S(t))$ has a compact global attractor if and only if it is asymptotically compact.

The main result of this paper is the following:

Theorem 2.4 Suppose that assumptions (2.2)-(2.5) hold. For $k, \beta>0$ such that

$$
\frac{1}{4}-\frac{3 k}{2 \lambda}-\frac{\beta}{2}>0
$$

the dynamical system $(\mathcal{H}, S(t))$ corresponding to system (1.6)-(1.11) has a compact global attractor $\mathcal{A} \subset \mathcal{H}$.

\section{Global attractor}

To show Theorem 2.4, we apply the abstract results presented in the previous section. Accordingly, we shall first prove that the dynamical system $(\mathcal{H}, S(t))$ is dissipative. By Theorem 2.3 we need to verify the asymptotic compactness.

We have the following lemma on the system energy defined by

$$
\begin{aligned}
E(t)= & \frac{1}{2}\left\|u_{t}\right\|^{2}+\frac{1}{2}\|\Delta u\|^{2}+\frac{k}{2}\left\|u^{+}\right\|^{2}+\frac{1}{2}\|\theta\|^{2}+\frac{1}{2}\left\|\eta^{t}\right\|_{\mu, V_{2}}^{2}+\frac{1}{2}\left\|\zeta^{t}\right\|_{\nu, V_{1}}^{2} \\
& +\int_{\Omega}(F(u)-h u) d x .
\end{aligned}
$$

Lemma 3.1 Along the solution of (1.6)-(1.11), the energy E satisfies

$$
E^{\prime}(t)=-\left\|\nabla u_{t}\right\|^{2}-\|\nabla \theta\|^{2}+\frac{1}{2} \int_{0}^{\infty} \mu^{\prime}(s)\left\|\Delta \eta^{t}(s)\right\|^{2} d s+\frac{1}{2} \int_{0}^{\infty} v^{\prime}(s)\left\|\nabla \zeta^{t}(s)\right\|^{2} d s .
$$

Proof Multiplying equations (1.6) and (1.7) by $u_{t}$ and $\theta$, respectively, and integrating over $\Omega$, we get

$$
\begin{aligned}
& \frac{d}{d t}\left(\frac{1}{2}\left\|u_{t}\right\|^{2}+\frac{1}{2}\|\Delta u\|^{2}+\frac{k}{2}\left\|u^{+}\right\|^{2}+\frac{1}{2}\|\theta\|^{2}+\int_{\Omega}(F(u)-h u) d x\right) \\
& \quad+\left\|\nabla u_{t}\right\|^{2}+\|\nabla \theta\|^{2}+\left(\eta^{t}, u_{t}\right)_{\mu, V_{2}}+\left(\zeta^{t}, \theta\right)_{\nu, V_{1}}=0 .
\end{aligned}
$$

From (1.8) and (1.11) we have

$$
\left(\eta^{t}, u_{t}\right)_{\mu, V_{2}}=\left(\eta^{t}, \eta_{t}^{t}+\eta_{s}^{t}\right)_{\mu, V_{2}}=\frac{1}{2} \frac{d}{d t}\left\|\eta^{t}\right\|_{\mu, V_{2}}^{2}-\frac{1}{2} \int_{0}^{\infty} \mu^{\prime}(s)\left\|\Delta \eta^{t}(s)\right\|^{2} d s .
$$


Similarly, by (1.9) and (1.11) we obtain

$$
\left(\zeta^{t}, \theta\right)_{v, V_{1}}=\left(\zeta^{t}, \zeta_{t}^{t}+\zeta_{s}^{t}\right)_{v, V_{1}}=\frac{1}{2} \frac{d}{d t}\left\|\zeta^{t}\right\|_{\nu, V_{1}}^{2}-\frac{1}{2} \int_{0}^{\infty} v^{\prime}(s)\left\|\nabla \zeta^{t}(s)\right\|^{2} d s
$$

Combining (3.3) and (3.4) with (3.2), we get estimate (3.1).

To this system, we define the Lyapunov functional

$$
L(t)=M E(t)+\epsilon \phi(t)+\psi(t)
$$

with

$$
\begin{aligned}
& \phi(t)=\int_{\Omega} u_{t}(t) u(t) d x \\
& \psi(t)=-\int_{\Omega} u_{t}(t) \int_{0}^{\infty} \mu(s) \eta^{t}(s) d s d x-\int_{\Omega} \theta(t) \int_{0}^{\infty} v(s) \zeta^{t}(s) d s d x
\end{aligned}
$$

where $\epsilon>0$ and $M>0$ are to be fixed later.

Lemma 3.2 For $M>0$ sufficiently large, there exist positive constants $q_{0}, q_{1}$, and $C_{2}$ such that

$$
q_{0} E(t)-C_{2}|\Omega|-C_{2}\|h\|^{2} \leq L(t) \leq q_{1} E(t)+C_{2}|\Omega|+C_{2}\|h\|^{2}, \quad t \geq 0
$$

for any $0<\epsilon \leq 1$.

Proof The Young inequality, (2.1), and (2.3) give that

$$
\int_{\Omega}(F(u)-h u) d x \geq-k_{1}|\Omega|-\frac{1}{4}\|\Delta u\|^{2}-\frac{1}{\lambda}\|h\|^{2} .
$$

Then by energy and (3.6) we have

$$
\frac{1}{4}\left\|\left(u(t), u_{t}(t), \theta(t), \eta^{t}, \zeta^{t}\right)\right\|_{\mathcal{H}}^{2} \leq E(t)+k_{1}|\Omega|+\frac{1}{\lambda}\|h\|^{2} .
$$

From the Young inequality, (2.1), (2.4), and (3.7) we conclude that

$$
\begin{aligned}
|\phi(t)| & \leq \frac{1}{2}\left\|u_{t}\right\|^{2}+\frac{1}{2 \lambda}\|\Delta u\|^{2} \leq 2 \max \left\{1, \frac{1}{\lambda}\right\}\left(E(t)+k_{1}|\Omega|+\frac{1}{\lambda}\|h\|^{2}\right), \\
|\psi(t)| & \leq \frac{1}{2}\left\|u_{t}\right\|^{2}+\frac{1}{2}\|\theta\|^{2}+\frac{\mu_{0}}{2 \lambda}\left\|\eta^{t}\right\|_{\mu, V_{2}}^{2}+\frac{\nu_{0}}{2 \lambda_{1}}\left\|\zeta^{t}\right\|_{\nu, V_{1}}^{2} \\
& \leq 2 \max \left\{1, \frac{\mu_{0}}{\lambda}, \frac{\nu_{0}}{\lambda_{1}}\right\}\left(E(t)+k_{1}|\Omega|+\frac{1}{\lambda}\|h\|^{2}\right) .
\end{aligned}
$$

Choosing $C_{1}=2 \max \left\{1, \frac{1}{\lambda}, \frac{\mu_{0}}{\lambda}, \frac{\nu_{0}}{\lambda_{1}}\right\}$, for some $C_{2}>0$, we obtain

$$
|L(t)-M E(t)| \leq \epsilon|\phi(t)|+|\psi(t)| \leq C_{2}\left(E(t)+|\Omega|+\|h\|^{2}\right), \quad 0<\epsilon \leq 1 .
$$

Then, taking $M>C_{2}$, we get inequality (3.5) with $q_{0}=M-C_{2}$ and $q_{1}=M+C_{2}$. 
Lemma 3.3 We have the inequality

$$
\begin{aligned}
\phi^{\prime}(t) \leq & -E(t)-\left(\frac{1}{4}-\frac{3 k}{2 \lambda}-\frac{\beta}{2}\right)\|\Delta u\|^{2}+\frac{3}{2}\left\|u_{t}\right\|^{2}+\frac{1}{\lambda_{1} \beta}\left\|\nabla u_{t}\right\|^{2}+\frac{1}{2}\|\theta\|^{2}+\frac{\beta}{\lambda_{1}}\|\nabla \theta\|^{2} \\
& +\left(\mu_{0}+\frac{1}{2}\right)\left\|\eta^{t}\right\|_{\mu, V_{2}}^{2}+\frac{1}{2}\left\|\zeta^{t}\right\|_{\nu, V_{1}}^{2} .
\end{aligned}
$$

Proof Using (1.6) and (2.3) and subtracting and adding $E(t)$, we obtain

$$
\begin{aligned}
\phi^{\prime}(t)= & -\|\Delta u\|^{2}-\int_{\Omega} \nabla u_{t} \nabla u d x-k \int_{\Omega} u^{+} u d x-\int_{0}^{\infty} \mu(s)\left(\int_{\Omega} \Delta \eta^{t}(s) \Delta u d x\right) d s \\
& +\beta \int_{\Omega} \nabla \theta \nabla u d x-\int_{\Omega} f(u) u d x+\int_{\Omega} h u d x+\left\|u_{t}\right\|^{2} \\
\leq & -E(t)-\frac{1}{2}\|\Delta u\|^{2}+\frac{3}{2}\left\|u_{t}\right\|^{2}+\frac{k}{2}\left\|u^{+}\right\|^{2}+\frac{1}{2}\|\theta\|^{2}+\frac{1}{2}\left\|\eta^{t}\right\|_{\mu, V_{2}}^{2}+\frac{1}{2}\left\|\zeta^{t}\right\|_{\nu, V_{1}}^{2} \\
& -\int_{\Omega} \nabla u_{t} \nabla u d x-k \int_{\Omega} u^{+} u d x-\int_{0}^{\infty} \mu(s)\left(\int_{\Omega} \Delta \eta^{t}(s) \Delta u d x\right) d s \\
& +\beta \int_{\Omega} \nabla \theta \nabla u d x .
\end{aligned}
$$

Note that

$$
\left|-\int_{0}^{\infty} \mu(s)\left(\int_{\Omega} \Delta \eta^{t}(s) \Delta u d x\right) d s\right| \leq \frac{1}{4}\|\Delta u\|^{2}+\mu_{0}\left\|\eta^{t}\right\|_{\mu, V_{2}}^{2}
$$

by assumption (2.4). From (2.1), the Young inequality, and the inequality $\left|u^{+}\right| \leq|u|$ we see that

$$
\begin{aligned}
& \left|-\int_{\Omega} \nabla u_{t} \nabla u d x\right| \leq \frac{\beta}{4}\|\Delta u\|^{2}+\frac{1}{\lambda_{1} \beta}\left\|\nabla u_{t}\right\|^{2}, \\
& \left|\beta \int_{\Omega} \nabla \theta \nabla u d x\right| \leq \frac{\beta}{4}\|\Delta u\|^{2}+\frac{\beta}{\lambda_{1}}\|\nabla \theta\|^{2}, \\
& \left|-k \int_{\Omega} u^{+} u d x\right| \leq \frac{k}{\lambda}\|\Delta u\|^{2} .
\end{aligned}
$$

Substituting (3.10)-(3.13) into (3.9), we get estimate (3.8).

Lemma 3.4 There exist positive constants $C_{3}$ and $C_{4}$ such that

$$
\begin{aligned}
\psi^{\prime}(t) \leq & -\frac{\mu_{0}}{2}\left\|u_{t}\right\|^{2}-\frac{\nu_{0}}{2}\|\theta\|^{2}+\left(\delta+\frac{\delta k^{2}}{\lambda}+\delta k_{0} C_{s} C_{E}^{p}\right)\|\Delta u\|^{2}+\left(\delta+\delta \beta^{2}\right)\left\|\nabla u_{t}\right\|^{2} \\
& +\left(\delta+\delta \beta^{2}\right)\|\nabla \theta\|^{2}+C_{3}\left\|\eta^{t}\right\|_{\mu, V_{2}}^{2}+C_{4}\left\|\zeta^{t}\right\|_{v, V_{1}}^{2}+\frac{1}{2}\|h\|^{2} \\
& -\frac{\mu(0)}{2 \mu_{0} \lambda} \int_{0}^{\infty} \mu^{\prime}(s)\left\|\Delta \eta^{t}(s)\right\|^{2} d s-\frac{v(0)}{2 v_{0} \lambda_{1}} \int_{0}^{\infty} v^{\prime}(s)\left\|\nabla \zeta^{t}(s)\right\|^{2} d s
\end{aligned}
$$

where $C_{3}$ depends on $\mu_{0}, \lambda_{1}, \lambda$, and $\delta$, and $C_{4}$ depends on $v_{0}$ and $\delta$. 
Proof Taking the derivative of the function $\psi$ and using equations (1.6)-(1.9), we have

$$
\begin{aligned}
\psi^{\prime}(t)= & \int_{\Omega}\left(\Delta^{2} u-\Delta u_{t}+k u^{+}+\int_{0}^{\infty} \mu(s) \Delta^{2} \eta^{t}(s) d s+\beta \Delta \theta+f(u)-h\right) \\
& \times \int_{0}^{\infty} \mu(s) \eta^{t}(s) d s d x \\
& -\int_{\Omega}\left(\Delta \theta+\beta \Delta u_{t}+\int_{0}^{\infty} v(s) \Delta \zeta^{t}(s) d s\right) \int_{0}^{\infty} v(s) \zeta^{t}(s) d s d x \\
& -\int_{\Omega} u_{t} \int_{0}^{\infty} \mu(s)\left(u_{t}-\eta_{s}^{t}(s)\right) d s d x-\int_{\Omega} \theta \int_{0}^{\infty} v(s)\left(\theta-\zeta_{s}^{t}(s)\right) d s d x .
\end{aligned}
$$

From the Young inequality, (2.1), and (2.4) we derive that, for any $\delta>0$,

$$
\begin{aligned}
& \left|\int_{\Omega} \Delta u \int_{0}^{\infty} \mu(s) \Delta \eta^{t}(s) d s d x\right| \leq \delta\|\Delta u\|^{2}+\frac{\mu_{0}}{4 \delta}\left\|\eta^{t}\right\|_{\mu, V_{2}}^{2}, \\
& \left|\int_{\Omega} \nabla u_{t} \int_{0}^{\infty} \mu(s) \nabla \eta^{t}(s) d s d x\right| \leq \delta\left\|\nabla u_{t}\right\|^{2}+\frac{\mu_{0}}{4 \delta \lambda_{1}}\left\|\eta^{t}\right\|_{\mu, V_{2}}^{2}, \\
& \left|k \int_{\Omega} u^{+} \int_{0}^{\infty} \mu(s) \eta^{t}(s) d s d x\right| \leq \frac{\delta k^{2}}{\lambda}\|\Delta u\|^{2}+\frac{\mu_{0}}{4 \delta \lambda}\left\|\eta^{t}\right\|_{\mu, V_{2}}^{2}, \\
& \left|\int_{\Omega}\left(\int_{0}^{\infty} \mu(s) \Delta \eta^{t}(s) d s\right)^{2} d x\right| \leq \mu_{0}\left\|\eta^{t}\right\|_{\mu, V_{2}}^{2}, \\
& \left|-\beta \int_{\Omega} \nabla \theta \int_{0}^{\infty} \mu(s) \nabla \eta^{t}(s) d s d x\right| \leq \delta \beta^{2}\|\nabla \theta\|^{2}+\frac{\mu_{0}}{4 \delta \lambda_{1}}\left\|\eta^{t}\right\|_{\mu, V_{2}}^{2}, \\
& \left|-\int_{\Omega} h \int_{0}^{\infty} \mu(s) \eta^{t}(s) d s d x\right| \leq \frac{1}{2}\|h\|^{2}+\frac{\mu_{0}}{2 \lambda}\left\|\eta^{t}\right\|_{\mu, V_{2}}^{2} \cdot
\end{aligned}
$$

Using (2.2), (3.7), and the Sobolev embedding, since $E(t)$ is decreasing, we obtain

$$
\begin{aligned}
\left|\int_{\Omega} f(u) \int_{0}^{\infty} \mu(s) \eta^{t}(s) d s d x\right| & \leq \int_{\Omega} k_{0}\left(1+|u|^{p}\right)|u|\left|\int_{0}^{\infty} \mu(s) \eta^{t}(s) d s\right| d x \\
& \leq k_{0}\left(1+\|u\|_{2(p+1)}^{p}\right)\|u\|_{2(p+1)}\left\|\int_{0}^{\infty} \mu(s) \eta^{t}(s) d s\right\| \\
& \leq \delta k_{0} C_{s} C_{E}^{p}\|\Delta u\|^{2}+\frac{\mu_{0}}{4 \delta \lambda}\left\|\eta^{t}\right\|_{\mu, V_{2}}^{2}
\end{aligned}
$$

where $C_{E}=2\left(E(0)+k_{1}|\Omega|+\frac{1}{\lambda}\|h\|^{2}\right)^{1 / 2}$. Moreover, it follows that

$$
\begin{aligned}
\left|\int_{\Omega} u_{t} \int_{0}^{\infty} \mu(s) \eta_{s}^{t}(s) d s d x\right| & =\left|-\int_{\Omega} u_{t} \int_{0}^{\infty} \mu^{\prime}(s) \eta^{t}(s) d s d x\right| \\
& \leq \frac{\mu_{0}}{2}\left\|u_{t}\right\|^{2}-\frac{\mu(0)}{2 \mu_{0} \lambda} \int_{0}^{\infty} \mu^{\prime}(s)\left\|\Delta \eta^{t}(s)\right\|^{2} d s .
\end{aligned}
$$

Similarly, we find that, for any $\delta>0$,

$$
\begin{aligned}
& \left|\int_{\Omega} \nabla \theta \int_{0}^{\infty} v(s) \nabla \zeta^{t}(s) d s d x\right| \leq \delta\|\nabla \theta\|^{2}+\frac{v_{0}}{4 \delta}\left\|\zeta^{t}\right\|_{\nu, V_{1}}^{2}, \\
& \left|\beta \int_{\Omega} \nabla u_{t} \int_{0}^{\infty} v(s) \nabla \zeta^{t}(s) d s d x\right| \leq \delta \beta^{2}\left\|\nabla u_{t}\right\|^{2}+\frac{v_{0}}{4 \delta}\left\|\zeta^{t}\right\|_{\nu, V_{1}}^{2},
\end{aligned}
$$




$$
\begin{aligned}
& \left|\int_{\Omega}\left(\int_{0}^{\infty} v(s) \nabla \zeta^{t}(s) d s\right)^{2} d x\right| \leq v_{0}\left\|\zeta^{t}\right\|_{v, V_{1}}^{2}, \\
& \left|\int_{\Omega} \theta \int_{0}^{\infty} v(s) \zeta_{s}^{t}(s) d s d x\right| \leq \frac{\nu_{0}}{2}\|\theta\|^{2}-\frac{v(0)}{2 \nu_{0} \lambda_{1}} \int_{0}^{\infty} v^{\prime}(s)\left\|\nabla \zeta^{t}(s)\right\|^{2} d s .
\end{aligned}
$$

Inserting (3.16)-(3.27) into (3.15), we deduce that

$$
\begin{aligned}
\psi^{\prime}(t) \leq & -\frac{\mu_{0}}{2}\left\|u_{t}\right\|^{2}-\frac{\nu_{0}}{2}\|\theta\|^{2}+\left(\delta+\frac{\delta k^{2}}{\lambda}+\delta k_{0} C_{s} C_{E}^{p}\right)\|\Delta u\|^{2} \\
& +\left(\delta+\delta \beta^{2}\right)\left\|\nabla u_{t}\right\|^{2}+\left(\delta+\delta \beta^{2}\right)\|\nabla \theta\|^{2} \\
& +\left(\mu_{0}+\frac{\mu_{0}}{4 \delta}+\frac{\mu_{0}}{2 \lambda}+\frac{\mu_{0}}{2 \delta \lambda_{1}}+\frac{\mu_{0}}{2 \delta \lambda}\right)\left\|\eta^{t}\right\|_{\mu, V_{2}}^{2}+\left(v_{0}+\frac{v_{0}}{2 \delta}\right)\left\|\zeta^{t}\right\|_{\nu, V_{1}}^{2} \\
& +\frac{1}{2}\|h\|^{2}-\frac{\mu(0)}{2 \mu_{0} \lambda} \int_{0}^{\infty} \mu^{\prime}(s)\left\|\Delta \eta^{t}(s)\right\|^{2} d s-\frac{v(0)}{2 v_{0} \lambda_{1}} \int_{0}^{\infty} v^{\prime}(s)\left\|\nabla \zeta^{t}(s)\right\|^{2} d s .
\end{aligned}
$$

Lemma 3.5 Suppose that conditions (2.2)-(2.5) hold. Then the dynamical system $(\mathcal{H}, S(t))$ corresponding to problem (1.6)-(1.11) has a bounded absorbing set $\mathcal{B} \subset \mathcal{H}$.

Proof From (2.5), (3.1), (3.8), and (3.14) we see that

$$
\begin{aligned}
L^{\prime}(t) \leq & -\epsilon E(t)-\left(\frac{\mu_{0}}{2}-\frac{3 \epsilon}{2}\right)\left\|u_{t}\right\|^{2}-\left(\frac{\nu_{0}}{2}-\frac{\epsilon}{2}\right)\|\theta\|^{2} \\
& -\left(M-\frac{\epsilon}{\lambda_{1} \beta}-\left(1+\beta^{2}\right) \delta\right)\left\|\nabla u_{t}\right\|^{2}-\left(M-\frac{\beta \epsilon}{\lambda_{1}}-\left(1+\beta^{2}\right) \delta\right)\|\nabla \theta\|^{2} \\
& -\left[\left(\frac{1}{4}-\frac{3 k}{2 \lambda}-\frac{\beta}{2}\right) \epsilon-\left(1+\frac{k^{2}}{\lambda}+k_{0} C_{s} C_{E}^{p}\right) \delta\right]\|\Delta u\|^{2} \\
& +\left(\frac{M}{2}-\frac{\epsilon}{k_{2}}\left(\mu_{0}+\frac{1}{2}\right)-\frac{C_{3}}{k_{2}}-\frac{\mu(0)}{2 \mu_{0} \lambda}\right) \int_{0}^{\infty} \mu^{\prime}(s)\left\|\Delta \eta^{t}(s)\right\|^{2} d s \\
& +\left(\frac{M}{2}-\frac{\epsilon}{2 k_{3}}-\frac{C_{4}}{k_{3}}-\frac{v(0)}{2 v_{0} \lambda_{1}}\right) \int_{0}^{\infty} v^{\prime}(s)\left\|\nabla \zeta^{t}(s)\right\|^{2} d s+\frac{1}{2}\|h\|^{2} .
\end{aligned}
$$

We choose $\epsilon$ so small that

$$
\frac{\mu_{0}}{2}-\frac{3 \epsilon}{2}>0, \quad \frac{\nu_{0}}{2}-\frac{\epsilon}{2}>0
$$

For $k, \beta$ such that $\frac{1}{4}-\frac{3 k}{2 \lambda}-\frac{\beta}{2}>0$ and fixed $\epsilon$, we take $\delta>0$ small enough such that

$$
\left(\frac{1}{4}-\frac{3 k}{2 \lambda}-\frac{\beta}{2}\right) \epsilon-\left(1+\frac{k^{2}}{\lambda}+k_{0} C_{s} C_{E}^{p}\right) \delta>0
$$

Finally, we choose $M>0$ large enough such that

$$
\begin{aligned}
M> & \max \left\{\frac{\epsilon}{\lambda_{1} \beta}+\left(1+\beta^{2}\right) \delta, \frac{\beta \epsilon}{\lambda_{1}}+\left(1+\beta^{2}\right) \delta,\right. \\
& \left.\frac{\epsilon}{k_{2}}\left(2 \mu_{0}+1\right)+\frac{2 C_{3}}{k_{2}}+\frac{\mu(0)}{\mu_{0} \lambda}, \frac{\epsilon}{k_{3}}+\frac{2 C_{4}}{k_{3}}+\frac{\nu(0)}{v_{0} \lambda_{1}}\right\} .
\end{aligned}
$$


Then we deduce that

$$
L^{\prime}(t) \leq-\epsilon E(t)+\frac{1}{2}\|h\|^{2} .
$$

From (3.5) we get

$$
L^{\prime}(t) \leq-\frac{\epsilon}{q_{1}} L(t)+\frac{\epsilon C_{2}}{q_{1}}\left(|\Omega|+\|h\|^{2}\right)+\frac{1}{2}\|h\|^{2}
$$

which implies that

$$
\begin{aligned}
L(t) & \leq L(0) e^{-\frac{\epsilon}{q_{1}} t}+\left(\frac{\epsilon C_{2}}{q_{1}}+\frac{1}{2}\right)\left(|\Omega|+\|h\|^{2}\right) \int_{0}^{t} e^{-\frac{\epsilon}{q_{1}}(t-s)} d s \\
& =\left[L(0)-\left(C_{2}+\frac{q_{1}}{2 \epsilon}\right)\left(|\Omega|+\|h\|^{2}\right)\right] e^{-\frac{\epsilon}{q_{1}} t}+\left(C_{2}+\frac{q_{1}}{2 \epsilon}\right)\left(|\Omega|+\|h\|^{2}\right) .
\end{aligned}
$$

Using (3.5) again, we have

$$
E(t) \leq \frac{q_{1}}{q_{0}} E(0) e^{-\frac{\epsilon}{q_{1}} t}+\left(\frac{2 C_{2}}{q_{0}}+\frac{q_{1}}{2 \epsilon q_{0}}\right)\left(|\Omega|+\|h\|^{2}\right), \quad t \geq 0 .
$$

Consequently, (3.7) infers that

$$
\left\|\left(u(t), u_{t}(t), \theta(t), \eta^{t}, \zeta^{t}\right)\right\|_{\mathcal{H}}^{2} \leq C E(0) e^{-\frac{\epsilon}{q_{1}} t}+C\left(|\Omega|+\|h\|^{2}\right),
$$

where $C=4 \max \left\{\frac{q_{1}}{q_{0}}, \frac{2 C_{2}}{q_{0}}+\frac{q_{1}}{2 \epsilon q_{0}}+\frac{1}{\lambda}+k_{1}\right\}$ is a positive constant. Thus, taking the closed ball $\mathcal{B}=\bar{B}(0, R)$ with $R=\sqrt{2 C\left(|\Omega|+\|h\|^{2}\right)}$, we conclude from (3.28) that $\mathcal{B}$ is a bounded absorbing set of $(\mathcal{H}, S(t))$.

Lemma 3.6 Under the hypotheses of Theorem 2.4, given a bounded set $B \subset \mathcal{H}$, let $z_{1}=$ $\left(u, u_{t}, \theta, \eta, \zeta\right)$ and $z_{2}=\left(\tilde{u}, \tilde{u}_{t}, \tilde{\theta}, \tilde{\eta}, \tilde{\zeta}\right)$ be two weak solutions of system (1.6)-(1.11) with corresponding initial conditions $z_{1}(0)=\left(u_{0}, u_{1}, \theta_{0}, \eta_{0}, \zeta_{0}\right)$ and $z_{2}(0)=\left(\tilde{u}_{0}, \tilde{u}_{1}, \tilde{\theta}_{0}, \tilde{\eta}_{0}, \tilde{\zeta}_{0}\right) \in B$. Then there exist positive constants $\gamma, \tilde{C}_{0}$, and $\tilde{C}_{1}$ depending on $B$ such that

$$
\begin{aligned}
\left\|z_{1}(t)-z_{2}(t)\right\|_{\mathcal{H}}^{2} \leq & \tilde{C}_{0} e^{-\gamma t}\left\|z_{1}(0)-z_{2}(0)\right\|_{\mathcal{H}}^{2} \\
& +\tilde{C}_{1} \int_{0}^{t} e^{-\gamma(t-s)}\|u(s)-\tilde{u}(s)\|_{2(p+1)}^{2} d s, \quad t \geq 0 .
\end{aligned}
$$

Proof We set $w=u-\tilde{u}, \vartheta=\theta-\tilde{\theta}, \xi=\eta-\tilde{\eta}$, and $\tau=\zeta-\tilde{\zeta}$. Then $\left(w, w_{t}, \vartheta, \xi, \tau\right)$ is a weak solution of

$$
\begin{aligned}
& w_{t t}+\Delta^{2} w-\Delta w_{t}+k u^{+}-k \tilde{u}^{+}+\int_{0}^{\infty} \mu(s) \Delta^{2} \xi^{t}(s) d s+\beta \Delta \vartheta+f(u)-f(\tilde{u})=0, \\
& \vartheta_{t}-\Delta \vartheta-\beta \Delta w_{t}-\int_{0}^{\infty} v(s) \Delta \tau^{t}(s) d s=0 \\
& \xi_{t}=-\xi_{s}+w_{t} \\
& \tau_{t}=-\tau_{s}+\vartheta
\end{aligned}
$$


with initial conditions

$$
\begin{aligned}
& w(0)=u_{0}-\tilde{u}_{0}, \quad w_{t}(0)=u_{1}-\tilde{u}_{1}, \quad \vartheta(0)=\theta_{0}-\tilde{\theta}_{0}, \\
& \xi^{0}=\eta_{0}-\tilde{\eta}_{0}, \quad \tau^{0}=\zeta_{0}-\tilde{\zeta}_{0} .
\end{aligned}
$$

Now we consider the energy functional

$$
\begin{aligned}
G(t)= & \frac{1}{2}\|\Delta w(t)\|^{2}+\frac{1}{2}\left\|w_{t}(t)\right\|^{2} \\
& +\frac{1}{2}\|\vartheta(t)\|^{2}+\frac{1}{2}\left\|\xi^{t}\right\|_{\mu, V_{2}}^{2}+\frac{1}{2}\left\|\tau^{t}\right\|_{\nu, V_{1}}^{2}, \quad t \geq 0 .
\end{aligned}
$$

Step 1. There exists a constant $C_{5}>0$ such that

$$
\begin{aligned}
G^{\prime}(t) \leq & -\left\|\nabla w_{t}\right\|^{2}-\|\nabla \vartheta\|^{2}+\frac{\delta_{0}}{2}\left\|w_{t}\right\|^{2}+C_{5}\|w\|_{2(p+1)}^{2} \\
& +\frac{1}{2} \int_{0}^{\infty} \mu^{\prime}(s)\left\|\Delta \xi^{t}(s)\right\|^{2} d s+\frac{1}{2} \int_{0}^{\infty} v^{\prime}(s)\left\|\nabla \tau^{t}(s)\right\|^{2} d s
\end{aligned}
$$

where $C_{5}$ depends on $k, k_{0}, \delta_{0}, c_{0}$, and $C_{B}$.

To show this, we multiply (3.30) by $w_{t}$ and (3.31) by $\vartheta$, respectively. Integrating and using (3.32) and (3.33), we obtain

$$
\begin{aligned}
G^{\prime}(t)= & -\left\|\nabla w_{t}\right\|^{2}-\|\nabla \vartheta\|^{2}-k \int_{\Omega}\left(u^{+}-\tilde{u}^{+}\right) w_{t} d x-\int_{\Omega}(f(u)-f(\tilde{u})) w_{t} d x \\
& +\frac{1}{2} \int_{0}^{\infty} \mu^{\prime}(s)\left\|\Delta \xi^{t}(s)\right\|^{2} d s+\frac{1}{2} \int_{0}^{\infty} v^{\prime}(s)\left\|\nabla \tau^{t}(s)\right\|^{2} d s .
\end{aligned}
$$

By the Young inequality we get

$$
\begin{aligned}
\left|-k \int_{\Omega}\left(u^{+}-\tilde{u}^{+}\right) w_{t} d x\right| & \leq \frac{k^{2}}{\delta_{0}}\left\|u^{+}-\tilde{u}^{+}\right\|^{2}+\frac{\delta_{0}}{4}\left\|w_{t}\right\|^{2} \\
& \leq \frac{k^{2} c_{0}}{\delta_{0}}\|w\|_{2(p+1)}^{2}+\frac{\delta_{0}}{4}\left\|w_{t}\right\|^{2},
\end{aligned}
$$

where we have used the facts that $\left|u^{+}-\tilde{u}^{+}\right| \leq|u-\tilde{u}|$ and that $c_{0}>0$ is an embedding constant for $L^{2(p+1)}(\Omega) \hookrightarrow L^{2}(\Omega)$. In addition, from (2.2) and (3.28), by the generalized Hölder inequality with $\frac{p}{2(p+1)}+\frac{1}{2(p+1)}+\frac{1}{2}=1$ and the Young inequality we have

$$
\begin{aligned}
\left|-\int_{\Omega}(f(u)-f(\tilde{u})) w_{t} d x\right| & \leq k_{0} \int_{\Omega}\left(1+|u|^{p}+|\tilde{u}|^{p}\right)|w| \| w_{t} \mid d x \\
& \leq k_{0}\left(|\Omega|^{\frac{p}{2(p+1)}}+\|u\|_{2(p+1)}^{p}+\|\tilde{u}\|_{2(p+1)}^{p}\right)\|w\|_{2(p+1)}\left\|w_{t}\right\| \\
& \leq \frac{k_{0}^{2} C_{B}}{\delta_{0}}\|w\|_{2(p+1)}^{2}+\frac{\delta_{0}}{4}\left\|w_{t}\right\|^{2},
\end{aligned}
$$

where $C_{B}$ is a constant depending on $B$. Combining (3.37) and (3.38) with (3.36) we see that (3.35) holds. 
Step 2. Let us define the functional

$$
\Phi(t)=\int_{\Omega} w_{t}(t) w(t) d x
$$

Then there exists a constant $C_{6}>0$ such that

$$
\begin{aligned}
\Phi^{\prime}(t) \leq & -G(t)-\left(\frac{1}{4}-\frac{k}{\lambda}-\frac{\beta}{2}\right)\|\Delta w\|^{2}+\frac{3}{2}\left\|w_{t}\right\|^{2}+\frac{1}{\lambda_{1} \beta}\left\|\nabla w_{t}\right\|^{2}+\frac{1}{2}\|\vartheta\|^{2} \\
& +\frac{\beta}{\lambda_{1}}\|\nabla \vartheta\|^{2}+\left(2 \mu_{0}+\frac{1}{2}\right)\left\|\xi^{t}\right\|_{\mu, V_{2}}^{2}+\frac{1}{2}\left\|\tau^{t}\right\|_{\nu, V_{1}}^{2}+C_{6}\|w\|_{2(p+1)}^{2},
\end{aligned}
$$

where $C_{6}$ depends on $k_{0}, \lambda$, and $C_{B}$.

Indeed, differentiating the function $\Phi$, using (3.30), and adding and subtracting $G(t)$, we obtain

$$
\begin{aligned}
\Phi^{\prime}(t)= & -G(t)-\frac{1}{2}\|\Delta w\|^{2}+\frac{3}{2}\left\|w_{t}\right\|^{2}+\frac{1}{2}\|\vartheta\|^{2}+\frac{1}{2}\left\|\xi^{t}\right\|_{\mu, V_{2}}^{2}+\frac{1}{2}\left\|\tau^{t}\right\|_{v, V_{1}}^{2} \\
& -k \int_{\Omega}\left(u^{+}-\tilde{u}^{+}\right) w d x+\int_{\Omega} \Delta w_{t} w d x-\int_{\Omega} \int_{0}^{\infty} \mu(s) \Delta \xi^{t}(s) d s \Delta w d x \\
& -\beta \int_{\Omega} \Delta \vartheta w d x-\int_{\Omega}(f(u)-f(\tilde{u})) w d x .
\end{aligned}
$$

By a similar procedure used in Step 1, from (2.1), (2.2), (2.4), and the Young inequality we derive the following estimates:

$$
\begin{aligned}
& \left|-k \int_{\Omega}\left(u^{+}-\tilde{u}^{+}\right) w d x\right| \leq k\|w\|^{2} \leq \frac{k}{\lambda}\|\Delta w\|^{2}, \\
& \left|-\int_{\Omega} \int_{0}^{\infty} \mu(s) \Delta \xi^{t}(s) d s \Delta w d x\right| \leq \frac{1}{8}\|\Delta w\|^{2}+2 \mu_{0}\left\|\xi^{t}\right\|_{\mu, V_{2}}^{2}, \\
& \left|-\int_{\Omega}(f(u)-f(\tilde{u})) w d x\right| \leq \frac{1}{8}\|\Delta w\|^{2}+\frac{2 k_{0}^{2} C_{B}}{\lambda}\|w\|_{2(p+1)}^{2}, \\
& \left|-\int_{\Omega} \nabla w_{t} \nabla w d x\right| \leq \frac{\beta}{4}\|\Delta w\|^{2}+\frac{1}{\lambda_{1} \beta}\left\|\nabla w_{t}\right\|^{2}, \\
& \left|\beta \int_{\Omega} \nabla \vartheta \nabla w d x\right| \leq \frac{\beta}{4}\|\Delta w\|^{2}+\frac{\beta}{\lambda_{1}}\|\nabla \vartheta\|^{2} .
\end{aligned}
$$

Substituting (3.41)-(3.45) into (3.40), we get

$$
\begin{aligned}
\Phi^{\prime}(t) \leq & -G(t)-\left(\frac{1}{4}-\frac{k}{\lambda}-\frac{\beta}{2}\right)\|\Delta w\|^{2}+\frac{3}{2}\left\|w_{t}\right\|^{2}+\frac{1}{\lambda_{1} \beta}\left\|\nabla w_{t}\right\|^{2}+\frac{1}{2}\|\vartheta\|^{2} \\
& +\frac{\beta}{\lambda_{1}}\|\nabla \vartheta\|^{2}+\left(2 \mu_{0}+\frac{1}{2}\right)\left\|\xi^{t}\right\|_{\mu, V_{2}}^{2}+\frac{1}{2}\left\|\tau^{t}\right\|_{\nu, V_{1}}^{2}+\frac{2 k_{0}^{2} C_{B}}{\lambda}\|w\|_{2(p+1)}^{2}
\end{aligned}
$$

Step 3. Let us define the functional

$$
\Psi(t)=-\int_{\Omega} w_{t}(t) \int_{0}^{\infty} \mu(s) \xi^{t}(s) d s d x-\int_{\Omega} \vartheta(t) \int_{0}^{\infty} v(s) \tau^{t}(s) d s d x .
$$


Then there exist constants $C_{7}, C_{8}$, and $C_{9}>0$ such that

$$
\begin{aligned}
\Psi^{\prime}(t) \leq & -\frac{\mu_{0}}{2}\left\|w_{t}\right\|^{2}-\frac{v_{0}}{2}\|\vartheta\|^{2}+\left(\delta_{1}+\frac{\delta_{1} k^{2}}{\lambda}\right)\|\Delta w\|^{2}+\left(\delta_{1}+\delta_{1} \beta^{2}\right)\left\|\nabla w_{t}\right\|^{2} \\
& +\left(\delta_{1}+\delta_{1} \beta^{2}\right)\|\nabla \vartheta\|^{2}+C_{7}\|w\|_{2(p+1)}^{2}+C_{8}\left\|\xi^{t}\right\|_{\mu, V_{2}}^{2}+C_{9}\left\|\tau^{t}\right\|_{v, V_{1}}^{2} \\
& -\frac{\mu(0)}{2 \mu_{0} \lambda} \int_{0}^{\infty} \mu^{\prime}(s)\left\|\Delta \xi^{t}(s)\right\|^{2} d s-\frac{v(0)}{2 v_{0} \lambda_{1}} \int_{0}^{\infty} v^{\prime}(s)\left\|\nabla \tau^{t}(s)\right\|^{2} d s
\end{aligned}
$$

where $C_{7}, C_{8}$, and $C_{9}$ depend on $\delta_{1}, \mu_{0}, v_{0}, \lambda_{1}, \lambda$, and $C_{B}$. To prove this, we observe that, by (3.30) and (3.31),

$$
\begin{aligned}
\Psi^{\prime}(t)= & \int_{\Omega}\left(\Delta^{2} w-\Delta w_{t}+k u^{+}-k \tilde{u}^{+}+\int_{0}^{\infty} \mu(s) \Delta^{2} \xi^{t}(s) d s+\beta \Delta \vartheta\right) \\
& \times \int_{0}^{\infty} \mu(s) \xi^{t}(s) d s d x \\
& +\int_{\Omega}(f(u)-f(\tilde{u})) \int_{0}^{\infty} \mu(s) \xi^{t}(s) d s d x-\int_{\Omega} w_{t} \int_{0}^{\infty} \mu(s) \xi_{t}^{t}(s) d s d x \\
& -\int_{\Omega}\left(\Delta \vartheta+\beta \Delta w_{t}+\int_{0}^{\infty} v(s) \Delta \tau^{t}(s) d s\right) \int_{0}^{\infty} v(s) \tau^{t}(s) d s d x \\
& -\int_{\Omega} \vartheta \int_{0}^{\infty} v(s) \tau_{t}^{t}(s) d s d x
\end{aligned}
$$

Integrating with respect to $s$ and using (2.1), (2.4), (3.32), (3.33), and the Young inequality, we find that

$$
\begin{aligned}
-\int_{\Omega} w_{t} \int_{0}^{\infty} \mu(s) \xi_{t}^{t}(s) d s d x & =-\mu_{0}\left\|w_{t}\right\|^{2}-\int_{\Omega} w_{t} \int_{0}^{\infty} \mu^{\prime}(s) \xi^{t}(s) d s d x \\
& \leq-\frac{\mu_{0}}{2}\left\|w_{t}\right\|^{2}-\frac{\mu(0)}{2 \mu_{0} \lambda} \int_{0}^{\infty} \mu^{\prime}(s)\left\|\Delta \xi^{t}(s)\right\|^{2} d s
\end{aligned}
$$

and

$$
-\int_{\Omega} \vartheta \int_{0}^{\infty} v(s) \tau_{t}^{t}(s) d s d x \leq-\frac{\nu_{0}}{2}\|\vartheta\|^{2}-\frac{v(0)}{2 \nu_{0} \lambda_{1}} \int_{0}^{\infty} v^{\prime}(s)\left\|\nabla \tau^{t}(s)\right\|^{2} d s
$$

In addition, from (2.1), (2.4), and the Young inequality we have the following estimates for any $\delta_{1}>0$ :

$$
\begin{aligned}
& \left|\int_{\Omega} \Delta w \int_{0}^{\infty} \mu(s) \Delta \xi^{t}(s) d s d x\right| \leq \delta_{1}\|\Delta w\|^{2}+\frac{\mu_{0}}{4 \delta_{1}}\left\|\xi^{t}\right\|_{\mu, V_{2}}^{2}, \\
& \left|\int_{\Omega} \nabla w_{t} \int_{0}^{\infty} \mu(s) \nabla \xi^{t}(s) d s d x\right| \leq \delta_{1}\left\|\nabla w_{t}\right\|^{2}+\frac{\mu_{0}}{4 \delta_{1} \lambda_{1}}\left\|\xi^{t}\right\|_{\mu, V_{2}}^{2}, \\
& \left|k \int_{\Omega}\left(u^{+}-\tilde{u}^{+}\right) \int_{0}^{\infty} \mu(s) \xi^{t}(s) d s d x\right| \leq \frac{\delta_{1} k^{2}}{\lambda}\|\Delta w\|^{2}+\frac{\mu_{0}}{4 \delta_{1} \lambda}\left\|\xi^{t}\right\|_{\mu, V_{2}}^{2}, \\
& \left|\int_{\Omega}\left(\int_{0}^{\infty} \mu(s) \Delta \xi^{t}(s) d s\right)^{2} d x\right| \leq \mu_{0}\left\|\xi^{t}\right\|_{\mu, V_{2}}^{2},
\end{aligned}
$$




$$
\begin{aligned}
& \left|-\beta \int_{\Omega} \nabla \vartheta \int_{0}^{\infty} \mu(s) \nabla \xi^{t}(s) d s d x\right| \leq \delta_{1} \beta^{2}\|\nabla \vartheta\|^{2}+\frac{\mu_{0}}{4 \delta_{1} \lambda_{1}}\left\|\xi^{t}\right\|_{\mu, V_{2}}^{2}, \\
& \left|\int_{\Omega}(f(u)-f(\tilde{u})) \int_{0}^{\infty} \mu(s) \xi^{t}(s) d s d x\right| \leq \delta_{1} k_{0}^{2} C_{B}\|w\|_{2(p+1)}^{2}+\frac{\mu_{0}}{4 \delta_{1} \lambda}\left\|\xi^{t}\right\|_{\mu, V_{2}}^{2} .
\end{aligned}
$$

Moreover, we obtain that, for any $\delta_{1}>0$,

$$
\begin{aligned}
& \left|\int_{\Omega} \nabla \vartheta \int_{0}^{\infty} v(s) \nabla \tau^{t}(s) d s d x\right| \leq \delta_{1}\|\nabla \vartheta\|^{2}+\frac{v_{0}}{4 \delta_{1}}\left\|\tau^{t}\right\|_{v, V_{1}}^{2}, \\
& \left|\beta \int_{\Omega} \nabla w_{t} \int_{0}^{\infty} v(s) \nabla \tau^{t}(s) d s d x\right| \leq \delta_{1} \beta^{2}\left\|\nabla w_{t}\right\|^{2}+\frac{v_{0}}{4 \delta_{1}}\left\|\tau^{t}\right\|_{v, V_{1}}^{2}, \\
& \left|\int_{\Omega}\left(\int_{0}^{\infty} v(s) \nabla \tau^{t}(s) d s\right)^{2} d x\right| \leq v_{0}\left\|\tau^{t}\right\|_{\nu, V_{1}}^{2} .
\end{aligned}
$$

Therefore, we conclude that

$$
\begin{aligned}
\Psi^{\prime}(t) \leq & -\frac{\mu_{0}}{2}\left\|w_{t}\right\|^{2}-\frac{\nu_{0}}{2}\|\vartheta\|^{2}+\left(\delta_{1}+\frac{\delta_{1} k^{2}}{\lambda}\right)\|\Delta w\|^{2}+\left(\delta_{1}+\delta_{1} \beta^{2}\right)\left\|\nabla w_{t}\right\|^{2} \\
& +\left(\delta_{1}+\delta_{1} \beta^{2}\right)\|\nabla \vartheta\|^{2}+\left(\mu_{0}+\frac{\mu_{0}}{4 \delta_{1}}+\frac{\mu_{0}}{2 \delta_{1} \lambda_{1}}+\frac{\mu_{0}}{2 \delta_{1} \lambda}\right)\left\|\xi^{t}\right\|_{\mu, V_{2}}^{2} \\
& +\left(v_{0}+\frac{\nu_{0}}{2 \delta_{1}}\right)\left\|\tau^{t}\right\|_{\nu, V_{1}}^{2}+\delta_{1} k_{0}^{2} C_{B}\|w\|_{2(p+1)}^{2} \\
& -\frac{\mu(0)}{2 \mu_{0} \lambda} \int_{0}^{\infty} \mu^{\prime}(s)\left\|\Delta \xi^{t}(s)\right\|^{2} d s-\frac{v(0)}{2 v_{0} \lambda_{1}} \int_{0}^{\infty} v^{\prime}(s)\left\|\nabla \tau^{t}(s)\right\|^{2} d s .
\end{aligned}
$$

Step 4. We consider the functional

$$
\mathcal{G}(t)=N G(t)+\varepsilon \Phi+\Psi,
$$

where $\varepsilon \in(0,1)$ and $N>0$ are to be fixed later. Then there exists a constant $n_{0}>0$ such that, for $N>n_{0}$,

$$
n_{1} G(t) \leq \mathcal{G}(t) \leq n_{2} G(t), \quad t \geq 0
$$

where $n_{1}=N-n_{0}$ and $n_{2}=N+n_{0}$. Indeed, it is easy to see that

$$
\begin{aligned}
& |\Phi(t)| \leq \frac{1}{2}\left\|w_{t}\right\|^{2}+\frac{1}{2 \lambda}\|\Delta w\|^{2}, \\
& |\Psi(t)| \leq \frac{1}{2}\left\|w_{t}\right\|^{2}+\frac{1}{2}\|\vartheta\|^{2}+\frac{\mu_{0}}{2 \lambda}\left\|\xi^{t}\right\|_{\mu, V_{2}}^{2}+\frac{\nu_{0}}{2 \lambda_{1}}\left\|\tau^{t}\right\|_{\nu, V_{1}}^{2} .
\end{aligned}
$$

Therefore, choosing $n_{0}$ large enough, we get

$$
|\mathcal{G}(t)-N G(t)| \leq \varepsilon|\Phi(t)|+|\Psi(t)| \leq n_{0} G(t),
$$

and hence (3.48) holds. 
Step 5. From (2.5), (3.35), (3.39), and (3.47) we have

$$
\begin{aligned}
\mathcal{G}^{\prime}(t) \leq & -\varepsilon G(t)-\left(\frac{\mu_{0}}{2}-\frac{3 \varepsilon}{2}-\frac{\delta_{0} N}{2}\right)\left\|w_{t}\right\|^{2}-\left(\frac{\nu_{0}}{2}-\frac{\varepsilon}{2}\right)\|\vartheta\|^{2} \\
& -\left(N-\frac{\varepsilon}{\lambda_{1} \beta}-\left(1+\beta^{2}\right) \delta_{1}\right)\left\|\nabla w_{t}\right\|^{2}-\left(N-\frac{\beta \varepsilon}{\lambda_{1}}-\left(1+\beta^{2}\right) \delta_{1}\right)\|\nabla \vartheta\|^{2} \\
& -\left[\left(\frac{1}{4}-\frac{k}{\lambda}-\frac{\beta}{2}\right) \varepsilon-\left(1+\frac{k^{2}}{\lambda}\right) \delta_{1}\right]\|\Delta w\|^{2}+\left(C_{5} N+C_{6} \varepsilon+C_{7}\right)\|w\|_{2(p+1)}^{2} \\
& +\left(\frac{N}{2}-\left(2 \mu_{0}+\frac{1}{2}\right) \frac{\varepsilon}{k_{2}}-\frac{C_{8}}{k_{2}}-\frac{\mu(0)}{2 \mu_{0} \lambda}\right) \int_{0}^{\infty} \mu^{\prime}(s)\left\|\Delta \xi^{t}(s)\right\|^{2} d s \\
& +\left(\frac{N}{2}-\frac{\varepsilon}{2 k_{3}}-\frac{C_{9}}{k_{3}}-\frac{v(0)}{2 v_{0} \lambda_{1}}\right) \int_{0}^{\infty} v^{\prime}(s)\left\|\nabla \tau^{t}(s)\right\|^{2} d s .
\end{aligned}
$$

We first take $\varepsilon>0$ so small that

$$
\frac{\mu_{0}}{2}-\frac{3 \varepsilon}{2}>0, \quad \frac{\nu_{0}}{2}-\frac{\varepsilon}{2}>0 .
$$

For fixed $\varepsilon$, we choose $\delta_{1}>0$ so small that

$$
\left(\frac{1}{4}-\frac{k}{\lambda}-\frac{\beta}{2}\right) \varepsilon-\left(1+\frac{k^{2}}{\lambda}\right) \delta_{1}>0 .
$$

Next, for fixed $\delta_{1}$ and $\epsilon$, we take $N$ so large that

$$
\begin{aligned}
N> & \max \left\{\frac{\varepsilon}{\lambda_{1} \beta}+\left(1+\beta^{2}\right) \delta_{1}, \frac{\beta \varepsilon}{\lambda_{1}}+\left(1+\beta^{2}\right) \delta_{1},\right. \\
& \left.\left(4 \mu_{0}+1\right) \frac{\varepsilon}{k_{2}}+\frac{2 C_{8}}{k_{2}}+\frac{\mu(0)}{\mu_{0} \lambda}, \frac{\varepsilon}{k_{3}}+\frac{2 C_{9}}{k_{3}}+\frac{\nu(0)}{\nu_{0} \lambda_{1}}\right\} .
\end{aligned}
$$

Finally, choosing $\delta_{0}>0$ small enough, we get that there exist constants $\varepsilon_{0}, C_{10}>0$ such that

$$
\mathcal{G}^{\prime}(t) \leq-\varepsilon_{0} G(t)+C_{10}\|w\|_{2(p+1)}^{2}, \quad t \geq 0 .
$$

Combining (3.48) with (3.49), we obtain

$$
\mathcal{G}^{\prime}(t) \leq-\frac{\varepsilon_{0}}{n_{2}} \mathcal{G}(t)+C_{10}\|w\|_{2(p+1)}^{2}
$$

and so

$$
\mathcal{G}(t) \leq \mathcal{G}(0) e^{-\frac{\varepsilon_{0}}{n_{2}} t}+C_{10} \int_{0}^{t} e^{-\frac{\varepsilon_{0}}{n_{2}}(t-s)}\|w(s)\|_{2(p+1)}^{2} d s, \quad t \geq 0 .
$$

Using (3.48) again, we see that

$$
G(t) \leq \frac{n_{2}}{n_{1}} G(0) e^{-\frac{\varepsilon_{0}}{n_{2}} t}+\frac{C_{10}}{n_{1}} \int_{0}^{t} e^{-\frac{\varepsilon_{0}}{n_{2}}(t-s)}\|w(s)\|_{2(p+1)}^{2} d s, \quad t \geq 0 .
$$

Since $G(t)=\left\|z_{1}(t)-z_{2}(t)\right\|_{\mathcal{H}}^{2}$, we get (3.29) with $\tilde{C}_{0}=\frac{n_{2}}{n_{1}}, \gamma=\frac{\varepsilon_{0}}{n_{2}}$, and $\tilde{C}_{1}=\frac{C_{10}}{n_{1}}$. 
Using the ideas presented in $[25,26]$, we easily get the following lemma.

Lemma 3.7 Under the assumptions of Theorem 2.4, the dynamical system $(\mathcal{H}(t), S(t))$ corresponding to problem (1.6)-(1.11) is asymptotically smooth.

Proof Let $B$ be a bounded subset of $\mathcal{H}$ positively invariant with respect to $S(t)$. Denote by $C_{B}$ several positive constants that depend on $B$ but not on $t$. For $z_{1}^{0}, z_{2}^{0} \in B$, $S(t) z_{1}^{0}=\left(u(t), u_{t}(t), \theta(t), \eta^{t}, \zeta^{t}\right)$ and $S(t) z_{2}^{0}=\left(\tilde{u}(t), \tilde{u}_{t}(t), \tilde{\theta}(t), \tilde{\eta}^{t}, \tilde{\zeta}^{t}\right)$ are the solutions of (1.6)(1.9). Then, given $\epsilon>0$, by inequality (3.29) we can choose $T>0$ such that

$$
\left\|S(T) z_{1}^{0}-S(T) z_{2}^{0}\right\|_{\mathcal{H}} \leq \epsilon+C_{B}\left(\int_{0}^{T}\|u(s)-\tilde{u}(s)\|_{2(p+1)}^{2} d s\right)^{\frac{1}{2}},
$$

where $C_{B}>0$ is a constant depending only on the size of $B$. The condition $p>0$ implies that $2<2(p+1)<\infty$. Taking $\alpha_{0}=\frac{p}{2(p+1)}$ and applying the Gagliardo-Nirenberg interpolation inequality, we have

$$
\|u(t)-\tilde{u}(t)\|_{2(p+1)} \leq C\|\Delta(u(t)-\tilde{u}(t))\|^{\alpha_{0}}\|u(t)-\tilde{u}(t)\|^{1-\alpha_{0}} \leq C_{B}\|u(t)-\tilde{u}(t)\|^{1-\alpha_{0}} .
$$

Since $\|u(t)\|$ and $\|\tilde{u}(t)\|$ are uniformly bounded, there exists a constant $C_{B}>0$ such that

$$
\|u(t)-\tilde{u}(t)\|_{2(p+1)}^{2} \leq C_{B}\|u(t)-\tilde{u}(t)\|^{2\left(1-\alpha_{0}\right)}
$$

Therefore, from (3.50) and (3.51) we obtain

$$
\left\|S(T) z_{1}^{0}-S(T) z_{2}^{0}\right\|_{\mathcal{H}} \leq \epsilon+\Phi_{T}\left(z_{1}^{0}, z_{2}^{0}\right)
$$

with

$$
\Phi_{T}\left(z_{1}^{0}, z_{2}^{0}\right)=C_{B}\left(\int_{0}^{T}\|u(s)-\tilde{u}(s)\|^{2\left(1-\alpha_{0}\right)} d s\right)^{\frac{1}{2}} .
$$

Thus, by Theorem 2.2 it remains to prove that $\phi_{T}$ is a contractive function on $B \times B$. Indeed, given a sequence $\left(z_{n}^{0}\right)=\left(u_{n}^{0}, u_{n}^{1}, \theta_{n}^{0}, \eta_{n}^{0}, \zeta_{n}^{0}\right) \in B$, let us write $S(t)\left(z_{n}^{0}\right)=\left(u_{n}(t), u_{n, t}(t)\right.$, $\left.\theta_{n}(t), \eta_{n}^{t}, \zeta_{n}^{t}\right)$. Because $B$ is positively invariant by $S(t), t \geq 0$, it follows that the sequence $\left(u_{n}(t), u_{n, t}(t), \theta_{n}(t), \eta_{n}^{t}, \zeta_{n}^{t}\right)$ is uniformly bounded in $\mathcal{H}$. On the other hand,

$$
\left(u_{n}, u_{n, t}\right) \text { is bounded in } C\left([0, T], V_{2} \times V_{0}\right), \quad T>0 .
$$

By the compact embedding $V_{2} \subset V_{0}$ the Aubin lemma implies that there exists a subsequence $\left(u_{n_{k}}\right)$ that converges strongly in $C\left([0, T], V_{0}\right)$. Hence, we see that

$$
\lim _{k \rightarrow \infty} \lim _{l \rightarrow \infty} \int_{0}^{T}\left\|u_{n_{k}}(s)-u_{n_{l}}(s)\right\|^{2\left(1-\alpha_{0}\right)} d s=0 .
$$

This completes the proof of Lemma 3.7.

Proof of Theorem 2.4 From Lemmas 3.5 and 3.7 we conclude that $(\mathcal{H}, S(t))$ is a dissipative dynamical system, which is asymptotically smooth. Therefore, by Theorem 2.3 it has compact global attractor in $\mathcal{H}$. 


\section{Competing interests}

The author declares that she has no competing interests.

\section{Acknowledgements}

This research was supported by Basic Science Research Program through the National Research Foundation of Korea (NRF) funded by the Ministry of Science, ICT \& Future Planning (2014R1A1A1003440).

Received: 19 July 2016 Accepted: 8 November 2016 Published online: 24 November 2016

\section{References}

1. Lazer, AC, McKenna, PJ: Large-amplitude periodic oscillations in suspension bridge: some new connections with nonlinear analysis. SIAM Rev. 32, 537-578 (1990)

2. Ahmed, NU, Harbi, H: Mathematical analysis of dynamic models of suspension bridges. SIAM J. Appl. Math. 58(3), 853-874 (1998)

3. Litcanu, G: A mathematical of suspension bridge. Appl. Math. 49(1), 39-55 (2004)

4. An, Y: On the suspension bridge equations and the relevant problems. Doctoral thesis (2001)

5. Ma, QZ, Zhong, CK: Existence of global attractors for the suspension bridge equations. J. Sichuan Normal Univ. Nat. Sci. Ed. 43(2), 271-276 (2006)

6. Zhong, CK, Ma, QZ, Sun, CY: Existence of strong solutions and global attractors for the suspension bridge equations. Nonlinear Anal. 67, 442-454 (2007)

7. Park, JY, Kang, JR: Pullback $\mathcal{D}$-attractors for non-autonomous suspension bridge equations. Nonlinear Anal. 71, 4618-4623 (2009)

8. Park, JY, Kang, JR: Global attractors for the suspension bridge equations with nonlinear damping. Q. Appl. Math. 69, 465-475 (2011)

9. Ma, QZ, Zhong, CK: Existence of global attractors for the coupled system of suspension bridge equations. J. Math. Anal. Appl. 308, 365-379 (2005)

10. Ma, QZ, Zhong, CK: Existence of strong solutions and global attractors for the coupled suspension bridge equations. J. Differ. Equ. 246, 3755-3775 (2009)

11. Ma, QZ, Wang, BL: Existence of pullback attractors for the coupled suspension bridge equations. Electron. J. Differ. Equ. 2011, 16 (2011)

12. Kang, JR: Pullback attractors for the non-autonomous coupled suspension bridge equations. Appl. Math. Comput. 219, 8747-8758 (2013)

13. Kang, JR: Long-time behavior of a suspension bridge equations with past history. Appl. Math. Comput. 265, 509-519 (2015)

14. Ayadi, MA, Bchatnia, A, Hamouda, M, Messaoudi, S: General decay in a Timoshenko-type system with thermoelasticity with second sound. Adv. Nonlinear Anal. 4, 263-284 (2015)

15. Giorgi, C, Pata, V: Stability of abstract linear thermoelastic systems with memory. Math. Models Methods Appl. Sci. 11 627-644 (2001)

16. Grasselli, M, Rivera, JEM, Pata, V: On the energy decay of the linear thermoelastic plate with memory. J. Math. Anal. Appl. 309, 1-14 (2005)

17. Grasselli, M, Squassina, M: Exponential stability and singular limit for a linear thermoelastic plate with memory effects Adv. Math. Sci. Appl. 16, 15-31 (2006)

18. Dafermos, CM: Asymptotic stability in viscoelasticity. Arch. Ration. Mech. Anal. 37, 297-308 (1970)

19. Fabrizio, M, Giorgi, C, Pata, V: A new approach to equations with memory. Arch. Ration. Mech. Anal. 198, 189-232 (2010)

20. Giorgi, C, Rivera, JEM, Pata, V: Global attractors for a semilinear hyperbolic equation in viscoelasticity. J. Math. Anal. Appl. 260, 83-99 (2001)

21. Pata, V, Zucchi, A: Attractors for a damped hyperbolic equation with linear memory. Adv. Math. Sci. Appl. 11, 505-529 (2001)

22. Ma, QZ, Zhong, CK: Existence of strong global attractors for hyperbolic equation with linear memory. Appl. Math. Comput. 157, 745-758 (2004)

23. Park, JY, Kang, JR: Global attractor for hyperbolic equation with nonlinear damping and linear memory. Sci. China Math. 53(6), 1531-1539(2010)

24. Jorge Silva, MA, Ma, TF: On a viscoelastic plate equation with history setting and perturbation of $p$-Laplacian type. IMA J. Appl. Math. 78(6), 1130-1146 (2013)

25. Jorge Silva, MA, Ma, TF: Long-time dynamics for a class of Kirchhoff models with memory. J. Math. Phys. 54, 021505 (2013)

26. Oliveira Araújo, R, Ma, TF, Qin, YM: Long-time behavior of a quasilinear viscoelastic equation with past history. J. Differ. Equ. 254, 4066-4087 (2013)

27. Grasselli, M, Pata, V: Uniform attractors of nonautonomous dynamical systems with memory. Prog. Nonlinear Differ. Equ. Appl. 50, 155-178 (2002)

28. Temam, R: Infinite-Dimensional Dynamical Systems in Mechanics and Physics. Applied Mathematical Sciences, vol. 68. Springer, New York (1988)

29. Babin, AV, Vishik, MI: Attractors of Evolution Equations. Studies in Mathematics and Its Application, vol. 25 North-Holland, Amsterdam (1992)

30. Chueshov, I, Lasiecka, I: Von Karman Evolution Equations: Well-Posedness and Long-Time Dynamics. Springer Monographs in Mathematics. Springer, New York (2010)

31. Hale, JK: Asymptotic Behavior of Dissipative Systems. Mathematical Surveys and Monographs, vol. 25. American Mathematical Society, Providence (1988) 